\title{
Effet des variations de teneur en eau dans les sols compactés
}

\author{
Influence of moisture content variations in compacted soils
}

\author{
E. ALONSO \\ ETS Ingenieros de Caminos, Canales y Puertos Barcelone*
}

Rev. Franç. Géotech., n 62, pp. 7-21 (janvier 1993)

\section{Résumé}

La maîtrise de la teneur en eau de compactage est une préoccupation importante fondamentale des ingénieurs de génie civil. Aussi, la connaissance des principes fondamentaux du comportement des sols compactés est indispensable pour prévoir et quantifier les effets d'un changement de teneur en eau. Cet article, après avoir rappelé les phénomènes liés à la variation de la pression négative de l'eau, appelée succion, explique l'évolution de deux ouvrages réels, un remblaiement de carrière à ciel ouvert et un remblai de marnes compactées à partir des phénomènes analysés en laboratoire. Enfin, quelques conclusions concernant le comportement des sols non saturés soumis à une évolution de la teneur en eau sont présentées.

\begin{abstract}
Control of moisture content in compaction is an essential preoccupation for a civil engineer. Thus good knowledge of the basic principes behind the behaviour of compacted soils is vital when foreseeing and quantifying the influence of any change in moisture content. The present article first recalls the basic phenomena relating to any variation in negative water pressure, called suction, and then explains the evolution of two real-life fills, one a strip mining quarry and the other composed of compacted marl, on the basis of phenomena analysed in the laboratory. Finally, a few general conclusions are drawn concerning the behaviour of non saturated soils subjected to variations in moisture content.
\end{abstract}

- Departamento de Ingeniería del Terreno y Cartográfica, Universitat Politècnica de Catalunya C/Gran Capitán, s/n, Module D2, 08034 Barcelona, Espagne. 


\section{INTRODUCTION}

La sélection de matériaux pour la construction des rem. blais et les conditions de mise en œuvre sont en général réglementées par des normes qui attachent une importance spéciale à la maîtrise de la teneur en eau de compactage. L'objectif des recommandations de compactage est d'obtenir des structures stables et peu déformables au cours du temps. Cependant, lapplica. tion des spécifications n'est pas toujours respectée ou rigoureusement contrôlée sur certains chantiers. Cela souligne le jugement considérable nécessaire à l'ingénieur qui fixe ces spécifications. De plus, il n'est pas toujours possible de compter sur des matériaux de bonne qualité pour réaliser les remblais. En général, il faut les construire avec les matériaux disponibles à proximité du chantier.

Ainsi, la connaissance des principes fondamentaux qui gouvernent la réponse des remblais soumis à des variations de teneur en eau en place peut aider l'ingénieur responsable de l'opération à prendre la meilleure décision vis-à-vis du comportement présent ou futur.

Pour introduire le propos des prochains paragraphes, certains traits du comportement des sols compactés ont été introduits dans le tableau 1. Les phénomènes de déformation volumique $y$ sont surtout marqués quand le terrain s'humidifie après le compactage. Ceci est un phénomène fondamental. Dans les paragraphes sujvants d'autres questions seront examinées, telles que la dégradation et l'altération mécanique des matériaux et les distorsions causées par la déformation de cisaillement dans les talus.

Etant donné que les forces entre particules, causées par des contraintes extérieures (en particulier le poids du matériau) sont faibles, les forces d'origine capillaire ont une intensité comparable à celles-ci et jouent donc un rôle prépondérant. Une mesure globale de ces forces internes dans un sol compacté est l'intensité de la pres. sion (négative) de l'eau par rapport à la pression de Pair (en général atmosphérique).

Tableau 1. - Effet de l'eau dans les sols compactés. Table 1. - Influence of water in compacted soils.

\begin{tabular}{|c|c|}
\hline $\begin{array}{c}\text { Eléments } \\
\text { caractéristiques }\end{array}$ & $\begin{array}{c}\text { Variation } \\
\text { volumique }\end{array}$ \\
\hline $\begin{array}{l}\text { Forces intergranulaires } \\
\text { faibles (faibles contrain- } \\
\text { tes extérieures). } \\
\text { Forces capillaires entre } \\
\text { particules comparables } \\
\text { aux forces extérieures. } \\
\text { Interaction d'adsorption } \\
\text { eau-minéraux argileux. } \\
\text { Gonflement ou effondre- } \\
\text { ment après humidifica- } \\
\text { tion en fonction des } \\
\text { facteurs cités dans la co- } \\
\text { lonne de droite. }\end{array}$ & $\begin{array}{l}\text { La composition minéra- } \\
\text { logique. } \\
\text { La densité (ou l'indice } \\
\text { des vides) Yd (ou e) } \\
\text { (FONDAMENTAL). } \\
\text { La contrainte moyenne. } \\
\text { La teneur en eau initiale. } \\
\text { Le changement de suc- } \\
\text { cion. }\end{array}$ \\
\hline
\end{tabular}

Outre les contraintes totales d'origine "extérieure ", ce « défaut » de pression de l'eau ou succion est donc une pression dont il faut tenir compte. La présence de particules d'argile et leur affinité avec l'eau mobilisent les mécanismes de gonflement et de retrait provoqués par les cycles d'humidification et de séchage. En définitive, un sol compacté peut gonfler ou s'effondrer quand sa teneur en eau s'élève. Le signe de la déformation et son intensité dépendent de l'ensemble des facteurs in. diqués dans le tableau 1.

Afin d'analyser ces phénomènes, dans le deuxième paragraphe nous présentons une description du comportement de base des sols compactés qui est fondée sur les résultats fournis par les essais de laboratoire. En revanche, au paragraphe 3 nous examinons le comportement in situ à partir d'observations sur le terrain et le comportement mesuré en laboratoire. Enfin, nous résumons les conclusions les plus importantes.

\section{COMPORTEMENT DES SOLS COMPACTÉS SUITE AUX CHANGEMENTS DE TENEUR EN EAU}

La structure du sol après compactage et la minéralogie sont les variables fondamentales qui gouvernent le comportement vis-à-vis des changements de la teneur en eau. Les structures ouvertes, qui correspondent à des faibles densités, sont sujettes à l'effondrement quand les forces internes d'origine capillaire, capables de rigidifier le sol, diminuent, suite à une humidification. Ce sont surtout les sables argileux qui sont capables de maintenir des microstructures relativement ouvertes en état non saturé, très résistantes à l'action des contraintes extérieures. Cependant, la destruction des ménisques, des ponts et des agglomérats argileux entre grains, qui d'ordinaire accompagne un processus d'imbibition peut provoquer d'importantes réductions de volume. Si la plage des contraintes appliquées est vaste, on constate que l'effondrement après l'imbibition augmente avec la contrainte appliquée jusqu'à atteindre un maximum, puis diminue pour des contraintes de confinement su. périeures. $\mathrm{Ce}$ comportement est logique du point de vue physique: à une succion donnée, la contrainte croissante anivera à rompre la structure du sol, mais simultanément provoquera des états denses qui, progressivement, résisteront à d'autres imbibitions.

Les exemples qui montrent ce comportement en laboratoire sont abondants: MATYAS et RADHAKRISHNA, 1968: YUDHBIR, 1982 ; DELGADO, 1986. Parfois, ce maximum n'apparait pas dans la plage des densités, succions et charges étudiées. Par exemple, DAPENA (1989) présente des essais d'effondrement sur une argile compactée de faible plasticité, dont le taux de compactage variait entre $87 \%$ et $103 \%$ de l'optimum Proctor Normal ; ces essais montrent une augmentation toujours croissante de l'effondrement avec la contrainte appliquée. Cependant, à faible densité, l'effondrement maximum apparaît à de très faibles contraintes, de telle sorte qu'en pratique il diminue avec la contrainte appliquée.

Lorsque la fraction argileuse contient des minéraux actifs, les mécanismes de gonflement se déclenchent 
quand les échantillons s'humidifient. On constate que la contrainte appliquée inhibe progressivement l'expansion (JIMENEZ SALAS et SERRATOSA, 1953; BRACKLEY, 1975; JUSTO et al., 1984). Le gonflement diminue en première analyse, approximativement linéairement avec le logarithme de la contrainte de confinement appliquée

Un sol compacté, avec un état initial déterminé $\left(\gamma_{d}, w\right)$, peut, par conséquent, gonfler ou s'effondrer s'il est humidifié. C'est la contrainte de confinement existant avant le changement d'humidité qui contrôle le signe (et l'intensité) de la variation volumique. Ceci est représenté schématiquement sur la figure 1a. Cette figure montre qu'il existe une transition continue entre les phéno. mènes de gonflement et d'effondrement. La contrainte qui inhibe tout mouvement peut être une définition appropriée de la pression de gonflement.

Comme cela a été discuté plus haut, tout comportement identifié en laboratoire ne serait qu'une partie de la courbe a de la figure 1 . La courbe $1 \mathrm{~b}$ serait le propre des sols lâches sans minéraux expansifs. En revanche, la courbe $1 \mathrm{c}$ correspondrait à des sols sablo-argileux ou mi-sableux sans minéraux expansifs, compactés à des densités raisonnablement élevées (ce serait le cas de
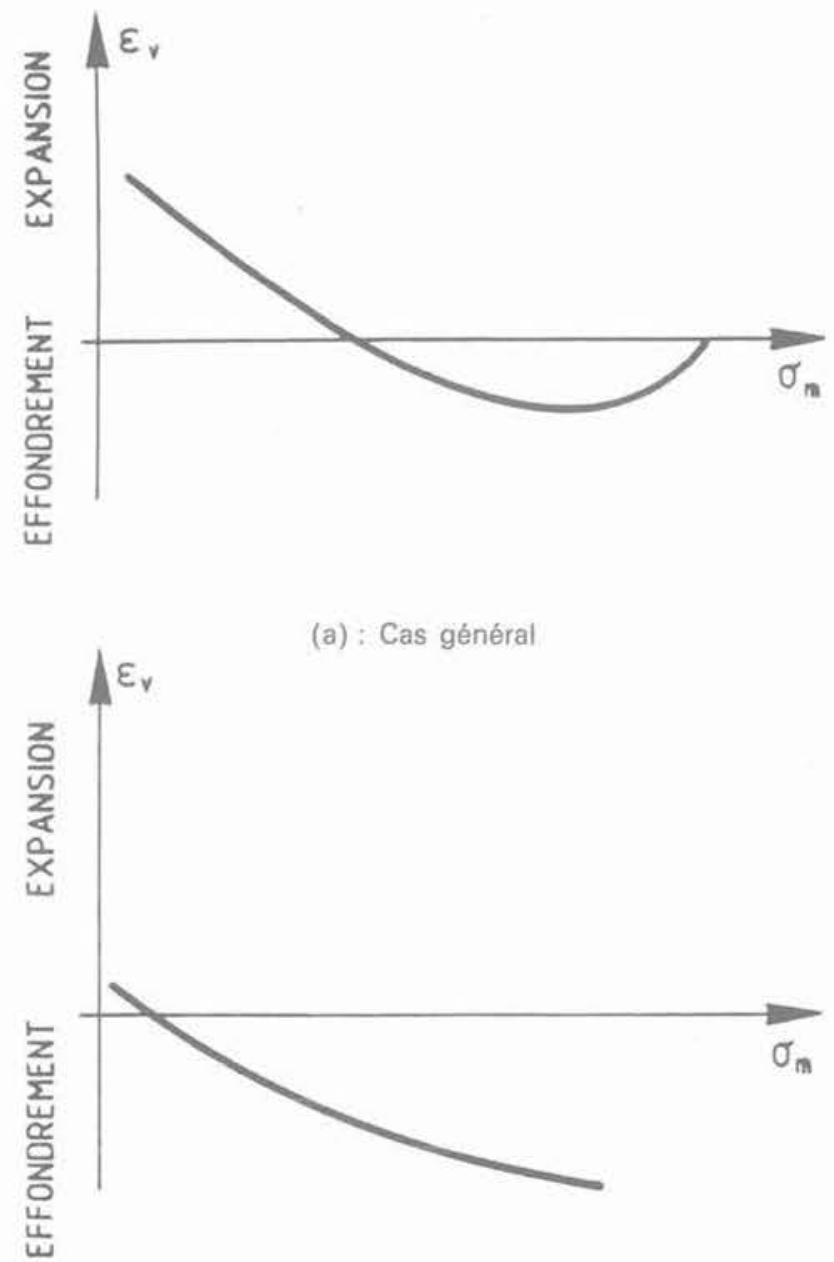

(c) : Sols bien compactés non-gonflants l'argile testée par DAPENA, 1989). Enfin, une argile gonflante ou de forte plasticité montrerait après compactage et humidification, pour un vaste éventail de contraintes, un comportement similaire à la courbe $1 \mathrm{~d}$.

Les équipements œdométriques à succion contrôlée (ESCARIO, 1967 ; BARDEN et al., 1969 ; KASSIF et BEN SHALOM, 1971; AITCHISON et MARTIN, 1973; FREDLUND et MORGENSTERN, 1976; COX, 1978; LLORET et ALONSO, 1985; VICOL, 1990; DELAGE et SURAJ DE SILVA, 1992, entre autres) ont permis de connaître plus profondément le processus de gonflement et d'effondrement au fur et à mesure de l'humidification du sol. Ainsi, il a été prouvé que les deux phénomènes: gonflement et effondrement de la structure peuvent avoir lieu simultanément, chacun avec une intensité différente. La déformation nette finale est le résultat des deux tendances. Des résultats de ce type ont été publiés par ESCARIO et SAEZ (1973) et COX (1978). Ce dernier a mené à terme une importante campagne d'essais œdométriques avec et sans contrôle de succion sur des échantillons de marne du Keupler prélevée dans un remblai d'autoroute. Sur la figure 2 sont reproduits des résultats de Cox obtenus en saturant sous une charge constante la marne compactée à une teneur en eau initiale constante mais à des densités

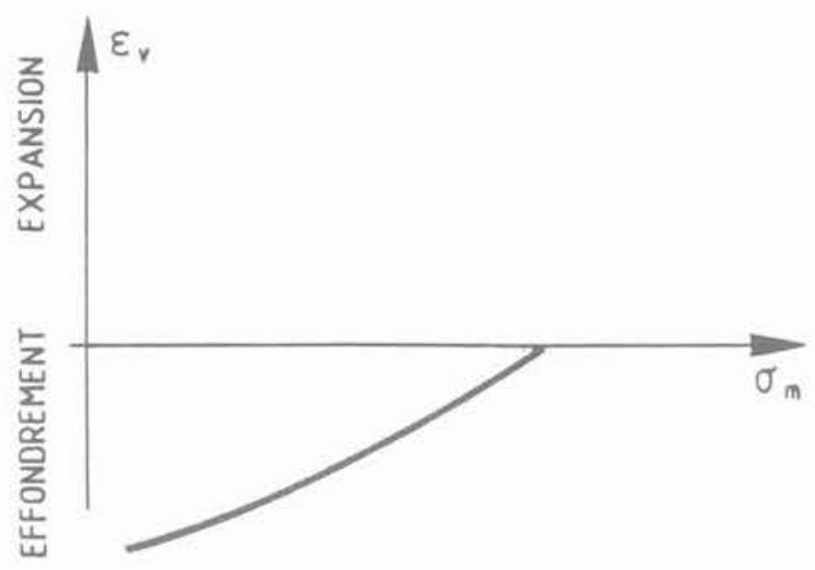

(b) : Sols non-gonflants à basse densité

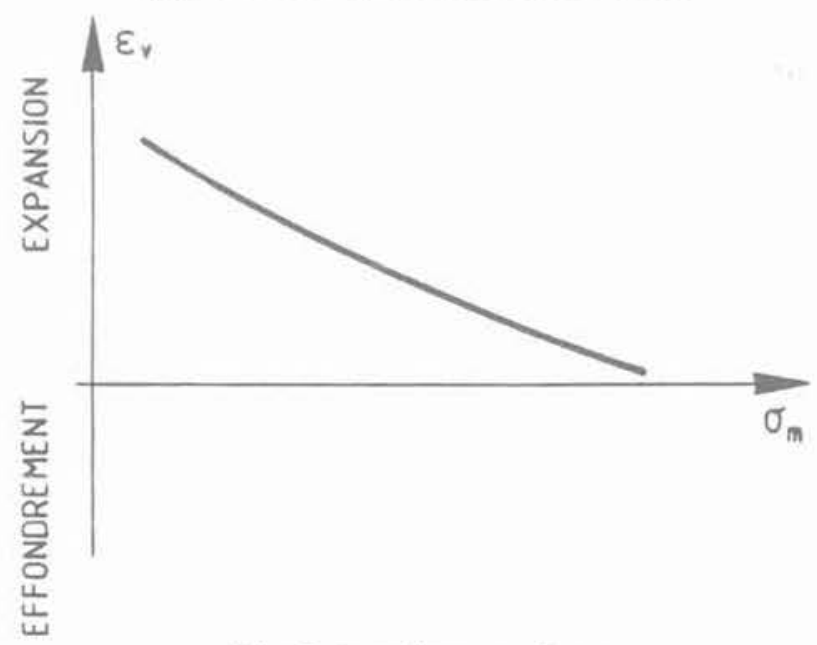

(d) : Sols argileux gonflants

Fig. 1 - Comportement volumique des sols non saturés quand ils sont humidifiés à une contrainte moyenne croissante. Fig. 1. - Volumetric behaviour of unsaturated soils subjected to wetting at increasing confining mean stress. 
différentes. On constate à quel point le gonflement, ou l'effondrement, varie au cours de ce processus d'humidification.

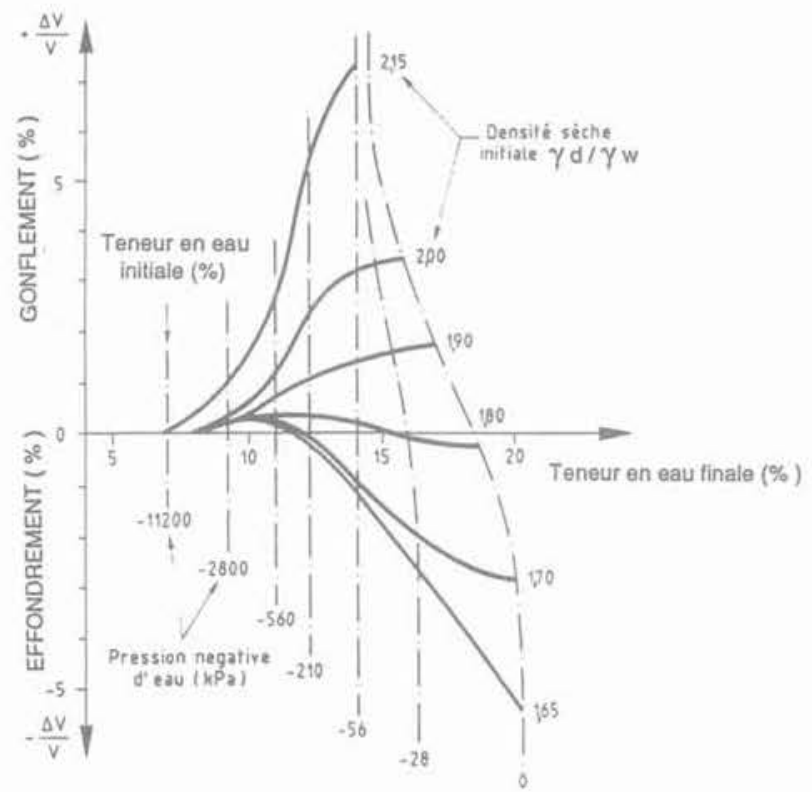

Fig. 2. - Déformations volumiques mesurées sur des échantillons de marne compactée à différentes densités pendant le processus de saturation (Cox, 1978).

Fig. 2. - Volumetric deformation measured during the saturation process on marl samples compacted at different densities (Cox, 1978)

En prévision de l'application de ces concepts il est utile de relier les déformations dues à la variation de teneur en eau aux variables de compactage, qui peuvent être contrôlées sur le chantier. Pour cela, il est préférable de partir de la représentation classique $(\gamma, w)$ où l'on peut situer les résultats de compactage à des énergies différentes. Si l'on réalise un nombre suffisant d'essais de gonflement-effondrement, sur cet espace on peut superposer une * carte * de déformations volumiques pour une contrainte de confinement donnée. Deux diagrammes de cette nature, correspondant à des conditions d'imbibition finale, ont été représentés dans la figure 3 (due à COX, 1978, pour la marne citée) et dans la figure 4 (ESCARIO, 1987, pour des matériaux de l'autoroute basco-aragonaise).

On observe sur ces deux figures l'influence dominante de la densité ou de l'indice des vides, atteinte après compactage. Sauf au voisinage de la courbe de saturation $(\mathrm{Sr}=1)$ les courbes d'égale expansion (ou d'égal effondrement) sont presque parallèles à l'axe des teneurs en eau. Cela permet, lorsque le sol n'est pas compacté en un état proche de la saturation, d'établir avec une bonne approximation une relation entre la variation de volume après l'imbibition et la densité après compactage (ou l'indice des vides) fonction de la contrainte de confinement. En première approximation, une relation de ce type serait valable pour des teneurs en eau inférieures à l'optimum Proctor. La figure 5 qui correspond à de l'ardoise compactée (ABEYESEKERA et al., 1978) montre une telle relation. On constate l'in.

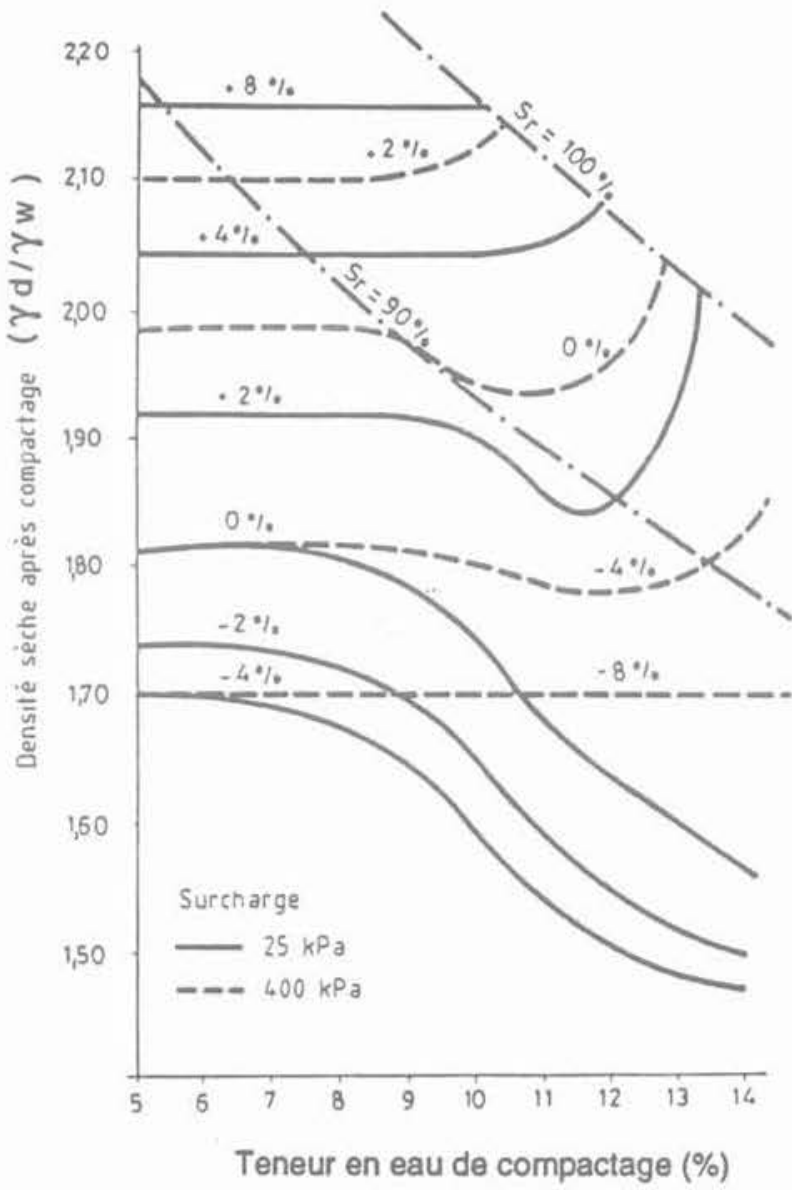

Fig. 3. - Gonflement ou effondrement après humidification en fonction de la teneur en eau et de la densité initiales (surcharge constante).

Fig. 3. - Swelling or collapse upon wetting as a

function of water content and initial dry density (constant load) (Cox, 1978).

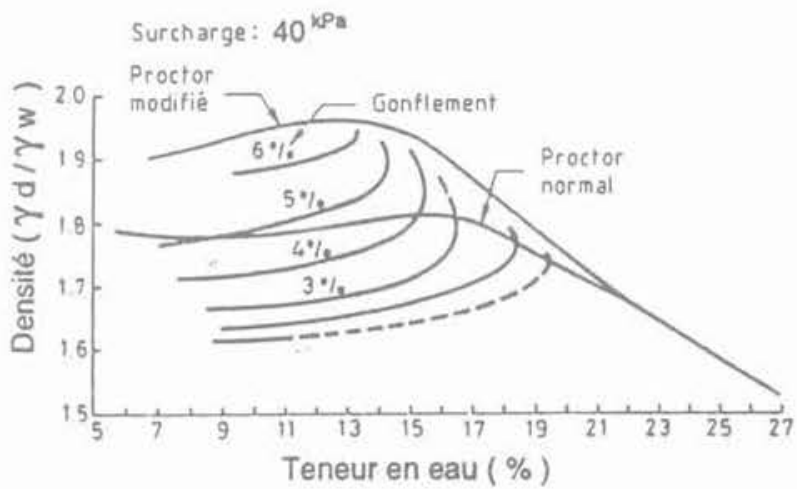

Fig. 4. - Courbes d'égal gonflement œodomètrique en fonction de la teneur en eau et de la densité initiales (Escario, 1987).

Fig. 4. - Curves of equal oedometric swelling as a function of water content and initial dry density (Escario, 1987).

fluence décisive de la densité initiale du matériau compacté sur son comportement ultérieur.

Il est clair aussi que la spécification d'une densité initiale déterminée, constante dans tout le remblai, n'empêche 
$\triangle$ Effondrement $(-)$

- Changement de volume nul

- Expansion (*)

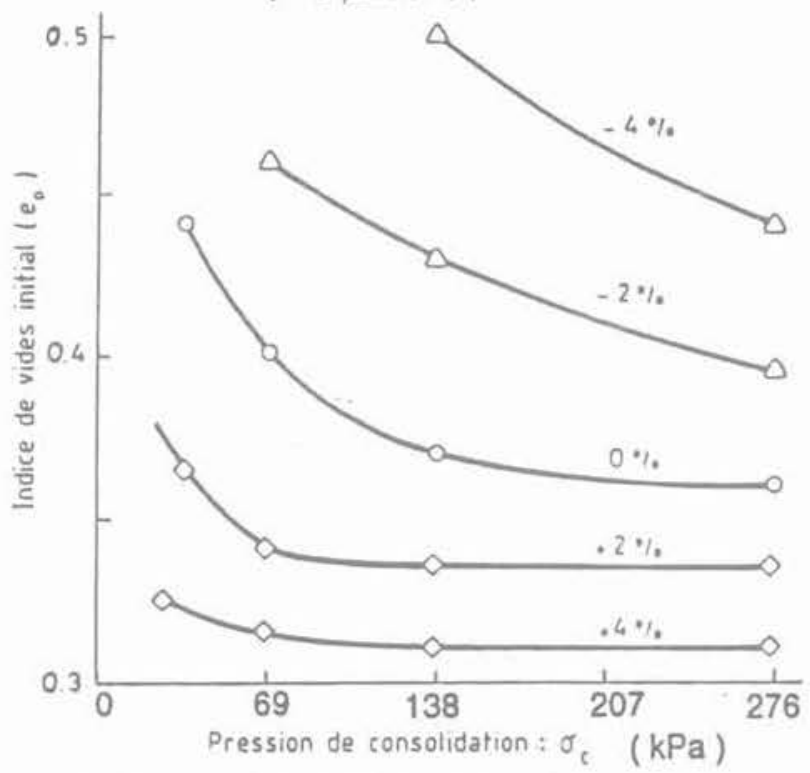

Fig. 5. - Tendance au changement de volume en humidifiant une ardoise compactée en fonction de l'indice des vides initial et de la pression de consolidation (Abeyesekera et al., 1978).

Fig. 5. - Volume change on wetting of a compacted shale as a function of initial void ratio and consolidation pressure (Abeyesekera et al., 1978)

pas les changements de volume. Afin de tendre vers une déformation volumique nulle d'un remblai, l'idéal serait de compacter plus énergiquement les couches profondes, et de moins compacter les couches super. ficielles. Même dans ce cas-là, on peut s'attendre à des mouvements, étant donné que la saturation n'atteint généralement pas l'ensemble du remblai, mais provoque une variation moins drastique de la succion au cours du temps, avec un comportement cyclique prépondé. rant, aux alentours de la surface exposée du terrain.

Bien qu'il soit difficile d'utiliser l'information fournie par des graphiques, tels que celui de la figure 5 , pour effectuer en pratique des prédictions quantitatives, ces ré. sultats peuvent être utiles pour avoir une idée du comportement que l'on peut espérer sur le chantier. ESCARIO (1987) citant TERZAGHI et PECK signale que des gonflements de plus de $5 \%$ sur des essais œedométriques après imbibition présagent d'un mauvais comportement prévisible au cours d'un remplissage. Cette valeur paraît très elevée. Dans le chapitre suivant on mentionne le cas d'un remblai de marnes compactées en France, ausculté avec précision, et qui a souffert d'importantes déformations au cours du temps. Dans ce cas-là alors que des poids spécifiques variant entre l'OPN et l'OPM ont été atteints sur le chantier, les gonflements maximaux mesurés en laboratoire (sous contrainte pratiquement nulle) varièrent entre 1,5 et $3 \%$.

\subsection{Effet des contraintes de cisaillement}

La grande majorité des résultats d'essais publiés ou pratiqués correspondent à des conditions œedométriques.
C'est le cas des figures 3,4 et 5 . Cependant, il paraît nécessaire de connaître le comportement des matériaux compactés sous des états plus généraux de contraintes et de succion. Plusieurs raisons peuvent être invoquées :

- des zones étendues de remblais sont faiblement confinées, dans des conditions très éloignées des conditions œdométriques. Dans ces parties (talus, couronnement) se situeront une bonne partie des éventuelles actions pathologiques d'un remblai : reptation des versants, glissements superficiels, fissuration et mouvernents différentiels des bords de la plate-forme, etc.

- les essais d'imbibition sous conditions œedométriques se trouvent loin des conditions de rupture.

En effet, des essais d'effondrement sous conditions $\mathrm{K}$ d'échantillons de sable argileux de faible plasticité ont été effectués par MASWOSWE (1985). Ces essais ont été réalisés à succion contrôlée dans une cellule triaxiale, de sorte que l'état des contraintes était connu. Les rêsultats d'un de ces essais sont reproduits sur la figure 6. L'échantillon a été soumis initialement à une augmentation de la contrainte verticale sans changement de teneur d'eau et postérieurement a été humidifié en maintenant la contrainte verticale constante, en empêchant les déformations latérales. Sur la figure $6 b$ apparaît le trajet de contraintes suivi par l'échantillon dans le plan ( $\mathrm{p} \cdot \mathrm{P}, \mathrm{q})$ et $(\mathrm{p} \cdot \mathrm{P}, \mathrm{q})$ où $\mathrm{p}=\left(\sigma_{1}+\sigma_{3}\right) /$ $2, \mathrm{q}=\left(\sigma_{1}-\sigma_{3}\right) / 2$, avec $\mathrm{P}$ et $\mathrm{P}$ qui sont respectivement les pressions d'air et d'eau. "On constate que le trajet de contraintes, après l'effondrement, se termine par l'état $\mathrm{K}$ correspondant au sol saturé. Les déformations volumiques mesurées au cours de l'essai sont indiquées sur la figure $6 \mathrm{a}$.

Afin d'analyser le comportement d'un sol compacté, lorsque des efforts de cisaillement sont susceptibles d'être signalés, au cours d'une évolution de la teneur en eau, nous décrirons brièvement un modèle constitutif, développé récemment et qui permet de reproduire les traits principaux du comportement de ces sols. Une description détaillée du modèle est rapportée par ALONSO, GENS et HIGHT (1987) et ALONSO, GENS et JOSA (1990).

Une idée fondarnentale du modèle est de considérer qu'il existe en cours de séchage une pression apparente de préconsolidation croissante avec la succion. Ceci se traduit par une plus grande "rigidité » du sol sous charges extérieures, situation largement vérifiable dans la pratique. Un autre aspect important de la formulation réside dans le choix des variables suivantes: l'excès de la contrainte moyenne sur la pression de l'air (p) et la succion ( $\mathrm{s}=\mathrm{P}-\mathrm{P}$ ) pour des conditions isotropes, auxquelles s'ajouterait la contrainte de cisaillement (q) pour la simulation des conditions triaxiales.

En partant, de la première idée, l'existence d'une courbe d'écrouissage, qui n'est autre que le lieu géornétrique des pressions de préconsolidation est proposée dans le plan $(p, s)$ en-deçà duquel se situeraient les états élastiques du sol (courbe LC) (fig. 7). Aussi bien un processus de charge à succion constante (L) à partir d'un état initial représenté par la pression de préconsolida. tion $\left(\mathrm{p}_{\mathrm{o}}\right)_{1}$ qu'un processus d'inondation à contrainte moyenne constante (trajectoire L) déplace la courbe d'écrouissage vers la droite en produisant en même temps des réductions de volume irréversibles. La courbe 


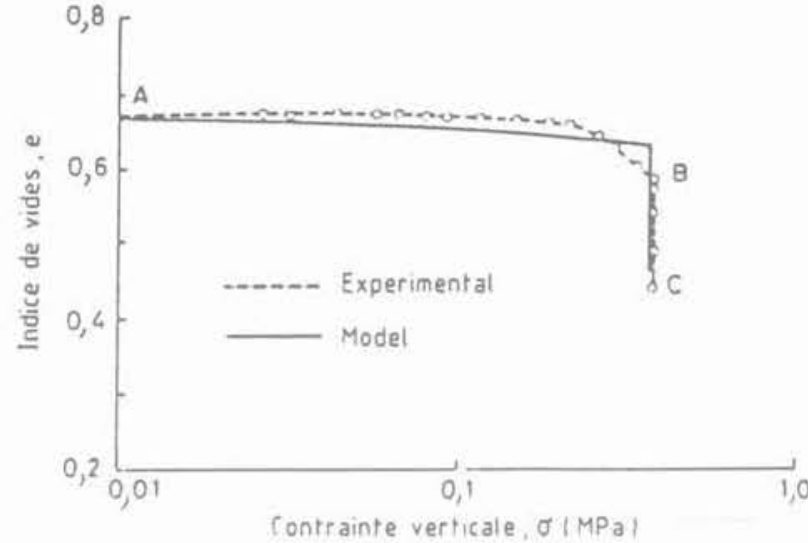

(a): Variation de l'indice des vides avec la contrainte verticale.

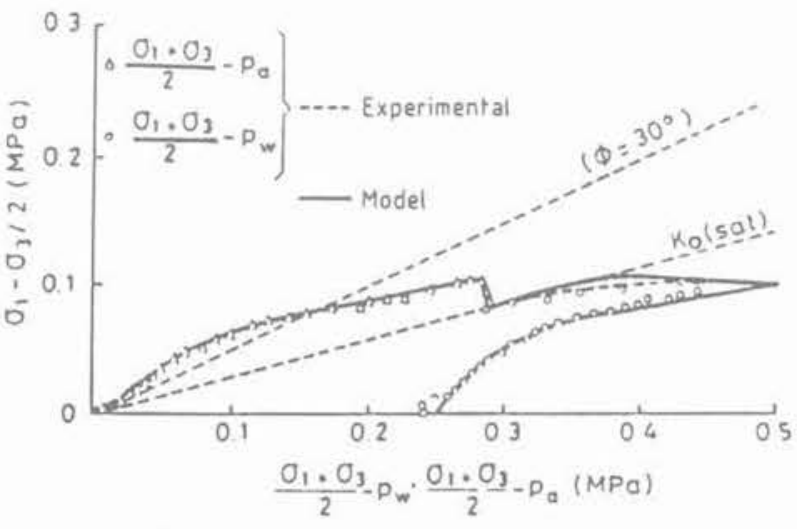

(b) : Chemin de contraintes dans le plan triaxial.

Fig. 6. - Résultats de l'essai SK3 publiés par Maswoswe (1985) et prédictions du modèle.

Fig. 6. - Results of test SK3, published by Maswoswe (1985) and model prediction.

initiale d'écrouissage $\left(A_{1}, B_{1}\right)$ se déplace jusqu'à $\left(A_{2}\right.$, $\left.\mathrm{B}_{2}\right)$. Une mesure de la déformation volumique plastique peut être la distance $\left(A_{1}, A_{2}\right)$, c'est-à-dire la différence entre les pressions de préconsolidation en état saturé $\left.\left[\left(\mathrm{p}_{\mathrm{o}}\right)_{2}\right)_{2}-\left(\mathrm{p}_{\mathrm{o}}\right)_{1}\right]$.

Afin de mieux comprendre les essais œdométriques, il faut envisager un troisième axe (q), car lors de ces essais les contraintes de cisaillement ne sont pas nulles. Les idées fondamentales antérieures peuvent être étendues à lespace $(\mathrm{p}, \mathrm{q}, \mathrm{s})$ en supposant que pour chaque valeur de la succion, il existe une enveloppe d'écrouissage, qui a été prise comme elliptique. De cette façon, la surface d'écrouissage dans les conditions triaxiales adopte la forme signalée dans la figure 8 . On peut observer que les ellipses successives, pour des succions croissantes, passent par l'enveloppe d'écrouissage des états isotropes, LC. De même on assigne au sol non saturé une cohésion, croissante avec la succion. Grâce à ce modèle nous avons reproduit les essais de compression suivie de saturation dans les conditions $\mathrm{K}$. de MASWOSWE. Un exemple de la capacité du modèle à décrire le comportement du sol est visible sur la figure 6 où l'on a superposé, aux résultats expérimentaux, les prédictions du modèle. Le modèle décrit a été modifié

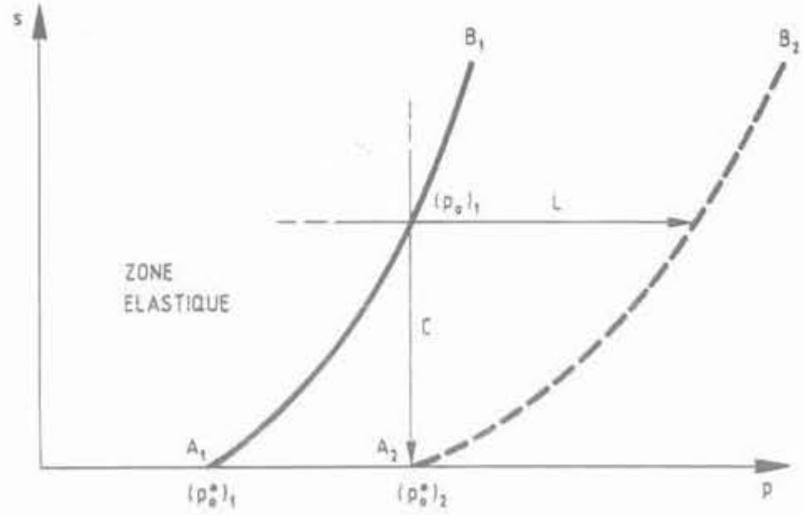

Fig. 7. - Courbes d'écrouissage pour décrire le comportement sous charge ( $L$ ) et l'effondrement (C) d'un sol partiellement saturé (Alonso, Gens et Hight, 1987).

Fig. 7. - Yield curves to explain the behaviour of a partially saturated soil under load (L) or collapse (C) (Alonso. Gens et Hight. 1987).

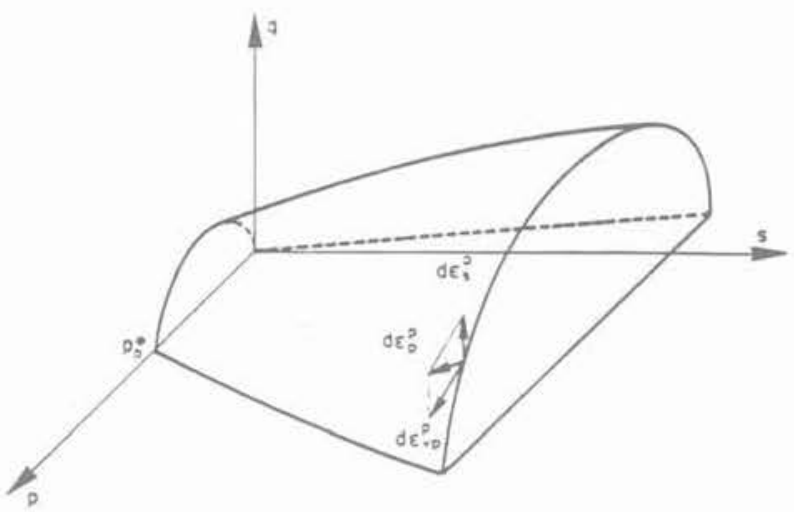

Fig. 8. - Surfaces d'écrouissage dans l'espace (p, q, s). Fig. 8. - Yield surface in the ( $p, q . s)$ space.

récemment afin de pouvoir simuler un maximum d'effondrement après l'inondation (BALMACEDA, 1991: JOSA et al., 1992). C'est avec ce nouveau modèle que les analyses indiquées plus bas ont été menées à bien.

Les conditions initiales des deux échantillons compactés, $\mathrm{A}$ et $\mathrm{B}$, analysées par MASWOSWE ont été représentées dans le diagramme $\left(\gamma_{\text {w }}, w\right)$ de la figure 9 , où est également reportée la courbe Proctor Normal. Les deux échantillons ont été compactês à des humidités proches de $w_{\text {OpN }}$ mais à des densités inférieures, surtout l'échantillon A. L'expérimentation détaillée de ces deux échantillons, menée par MASWOSWE, a permis d'identifier tous les paramètres du modèle de sorte que les trajectoires complètes de charge et d'effondrement dans des conditions $\mathrm{K}$ ont $\mathrm{pu}$ identifier la forme et la position des surfaces initiales d'écrouissage LC des deux sols ( $\mathrm{LC}_{\mathrm{tA}}$ et $\mathrm{LC}_{\mathrm{B}}$ à la figure 10). Le faible compactage de léchantillon $\mathrm{A}$ se traduit par de faibles valeurs de pression de préconsolidation apparente. Celles-ci sont beaucoup plus grandes sur l'échantillon $\mathrm{B}$, ( $\mathrm{LC}_{\mathrm{rp}}$ ) compacté plus énergiquement. Une fois «identifiées » les conditions de compactage par le biais du modèle, il est possible d'établir des comparaisons entre les deux en simulant des essais quelconques. Par 
exemple, la figure 10 représente les trajets de contrainte moyenne de confinement et de succion (p,s) que suivraient les deux échantillons soumis à un essai cedométrique à teneur en eau constante. La compression progressive des deux échantillons (non représentée) suppose une réduction du volume des pores et par conséquent une augmentation du degré de saturation. Ceci se traduit par une diminution de la succion comme on peut le constater sur les deux trajectoires dessinées. L'échantillon initialement plus compact (échantillon B) atteint évidemment plus vite la saturation totale $\left(S_{T}=1\right)$.

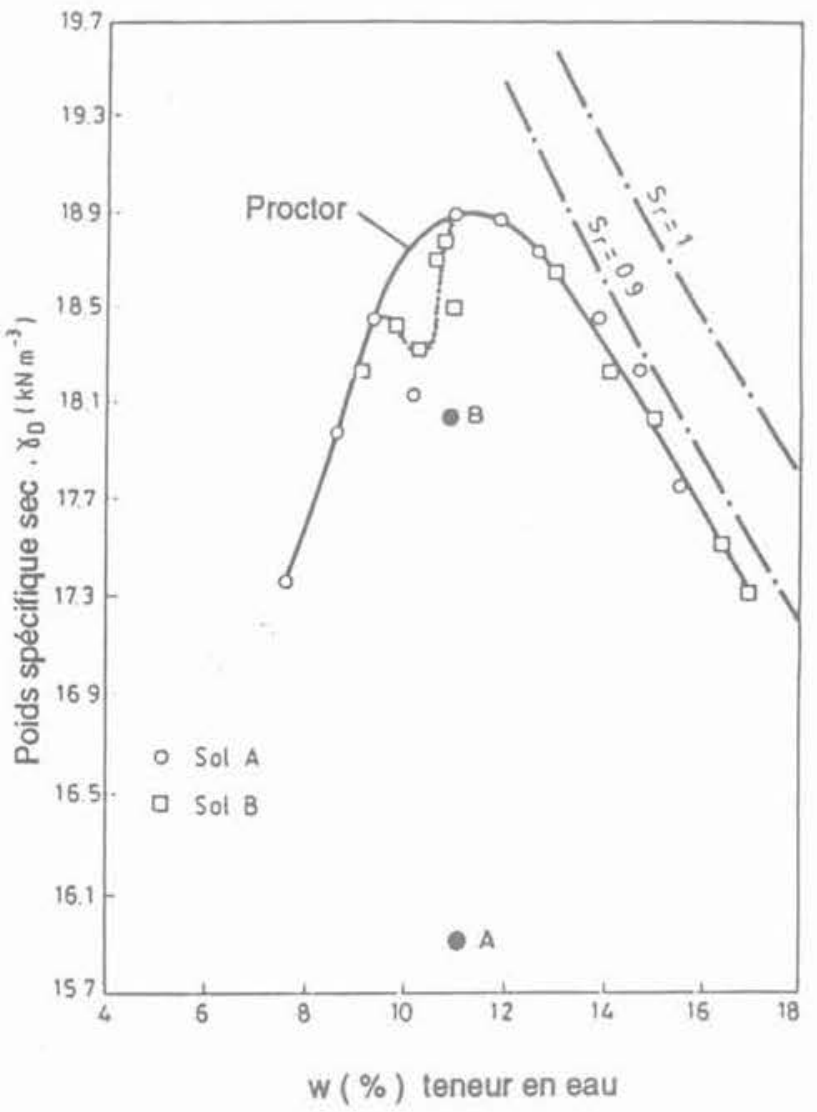

Fig. 9. - Conditions initiales de compactage des deux échantillons A et B analysés par Maswoswe (1985).

Fig. 9. - Initial compaction conditions of two samples. A and $B$, analyzed by Maswoswe (1985).

Il est aussi possible de simuler des essais d'inondation à partir de contraintes de confinement différentes. Afin de comparer le potentiel d'effondrement des deux échantillons, on a simulé des e essais d'effondrement " dans les positions indiquées sur la figure 10. Les résultats sont représentés sur la figure 11. L'échantillon $B$, plus dense, présente des gonflements pour des contraintes de confinement inférieures à environ $300 \mathrm{kPa}$ pour s'effondrer à des contraintes plus grandes. Les deux atteignent un effondrement maximum, qui diminue avec des contraintes de confinement croissantes.

Un modèle de ce type ouvre la possibilité d'effectuer des prédictions sans doute plus précises que les méthodes empiriques ou semi-empiriques. Mais il offre la

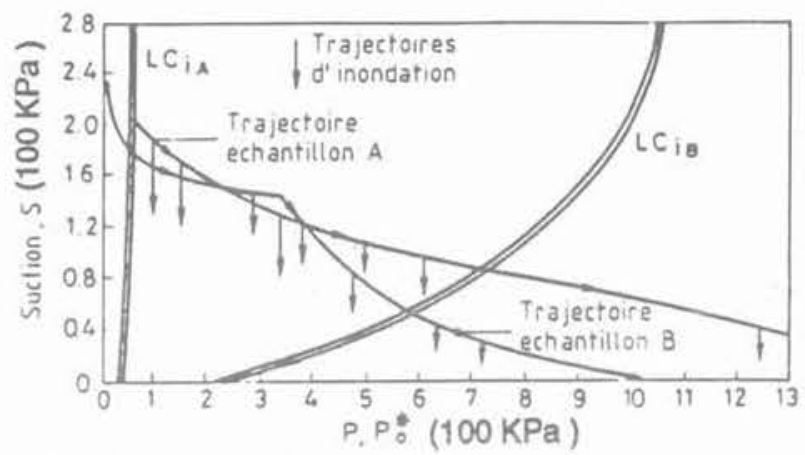

Fig. 10. - Surface initiale d'écrouissage et chemin des essais cedométriques des échantillons $A$ et $B$.

Fig. 10. - Initial yield curve and stress paths of oedometric tests on samples A and B.

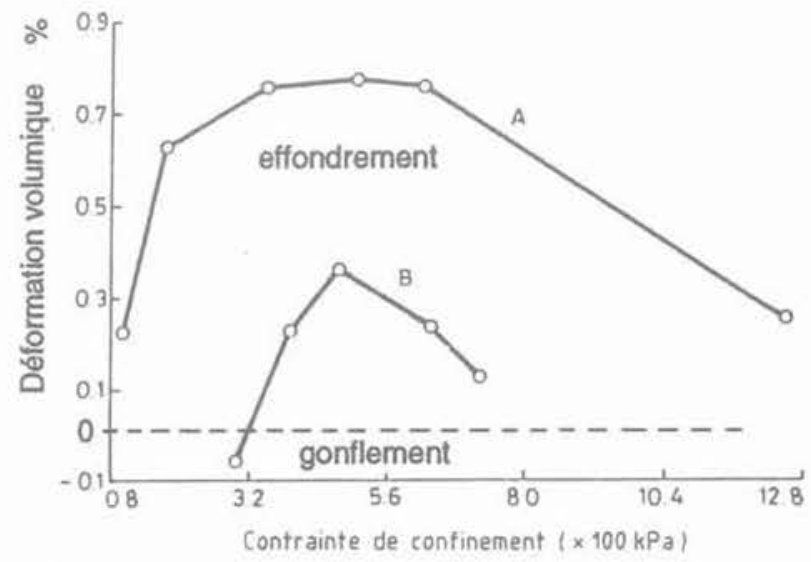

Fig. 11. - Effondrement calculé pour les échantillons $A$ et $B$ lors des essais cedométriques d'inondation sous contrainte.

Fig. 11. - Computed collapse of samples A and B during oedometric soaking tests under load.

possibilité, d'autant plus intéressante, de raisonner sur la réaction probable du terrain lors de changements de contrainte et de succion imposés par la construction et l'exploitation ultérieure des ouvrages en terre.

En effet, pendant la construction des remblais sont imposés au sol des trajets de contraintes complexes très éloignés des conditions œedométriques. Considérons, sur la figure $12 \mathrm{a}$, un point tel que A situé à proximité du talus d'un remblai. Le processus de compactage in. troduit des contraintes horizontales $\sigma_{h}$ plus grandes que les contraintes verticales $\sigma_{y}\left(q=\sigma_{v}-\sigma_{h}<0\right)$ (fig. 12c). Avec l'accumulation des couches les contraintes à forte composante verticale augmentent, tandis que la succion initiale diminue progressivement du fait que la porosité diminue. L'infiltration des eaux de pluie peut en dernier lieu réduire la succion sans changer considérablement l'état des contraintes totales. Dans un espace $(\mathrm{p}, \mathrm{q}, \mathrm{s})$ on a tracé la trajectoire probable de succion du point A (fig. 12d). La question consiste à évaluer l'effet que peut avoir l'humidification du sol dans ces conditions-là. Elle concerne le cas de vastes zones de talus de remblais soumises à des infiltrations, à des phénomènes d'ascension capillaire ou 

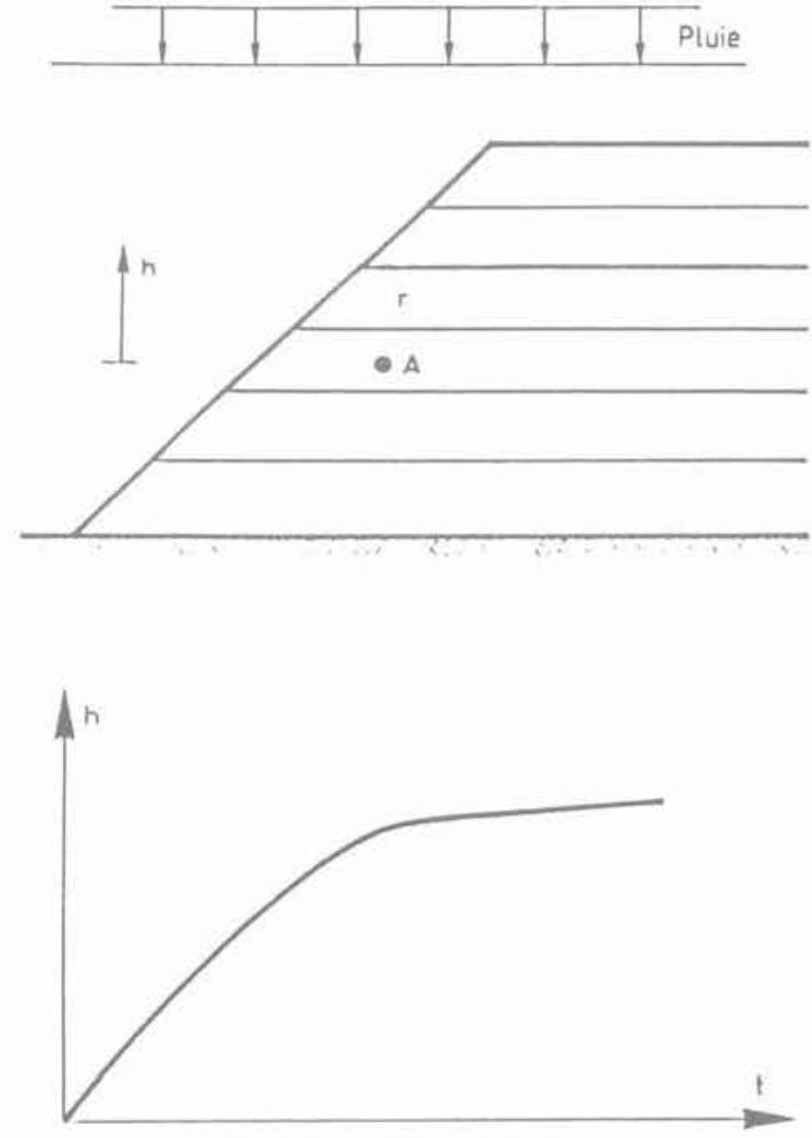

(a): Chemin de contraintes.

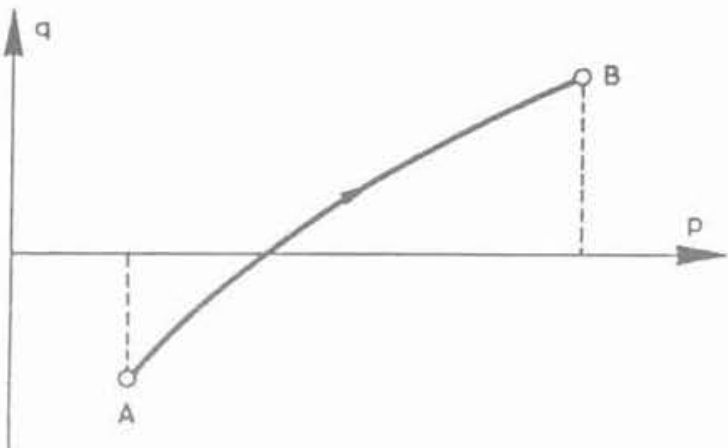

(b) : Augmentation de la hauteur du remblai.

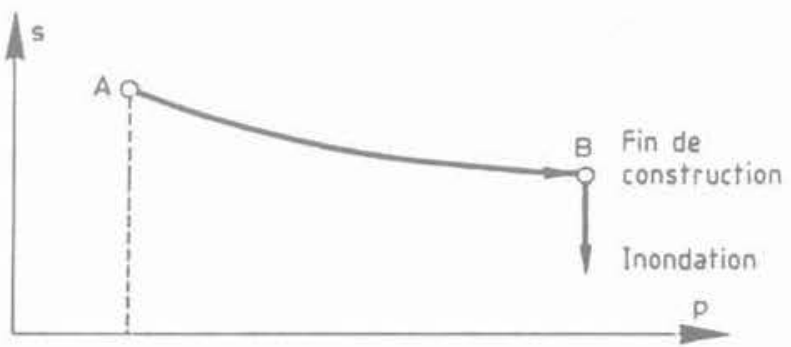

(c) : Trajectoire dans le plan (q, p).

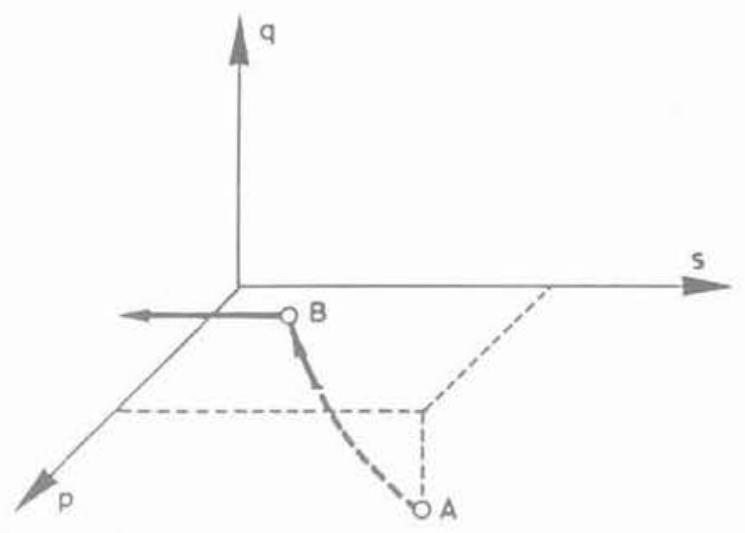

(d): Trajectoire dans le plan (p, s).

Fig. 12. - Chemin de contraintes probable d'un point $A$ aux alentours immédiats d'un talus en construction. Fig. 12. Expected stress path for a point $A$ in the vicinity of the slope of an embankment under construction.

d'infiltration par accumulation d'eau provenant d'un mauvais drainage ou de la rupture d'une conduite.

L'effet d'un tel chemin sur un sol non saturé a été représenté sur le figure 13. A partir d'un état initial, donné par une contrainte moyenne et une contrainte de cisaillement (point D de la figure 13a), on mouille le sol (trajectoire DE). L'analuse du comportement au cisaillement (fig. 13b) montre laccumulation des distorsions dans le sol qui peut se rompre, comme dans le cas représenté, si la réduction de succion est suffisam. ment élevée.

Dans certains cas il faudrait ajouter au mécanisme décrit la dégradation des propriétés du terrain compacté par effet de l'humidification. Ces deux phénomènes tendent à favoriser les déformations de cisaillement, la reptation dans les talus et le couronnement des remblais.

\section{OBSERVATIONS SUR LE TERRAIN}

Afin de mettre en évidence certains comportements caractéristiques des remblais, nous décrivons brièvement ci-dessous deux cas réels, relativement récents, caractéristiques en raison des mesures assez détaillées qui furent menées à terme. Ceci permet d'obtenir une image bien documentée du comportement réel. Le pre. mier se rapporte au rechargement hydraulique de remplissages de carrière, en général peu compactés; et le second au comportement, au cours de nombreuses années, d'un remblai de marnes argileuses compactées.

Le premier cas peut être représentatif du comportement de matériaux hétérogènes peu compactés et le second de remblais construits avec des matériaux évolutifs. 


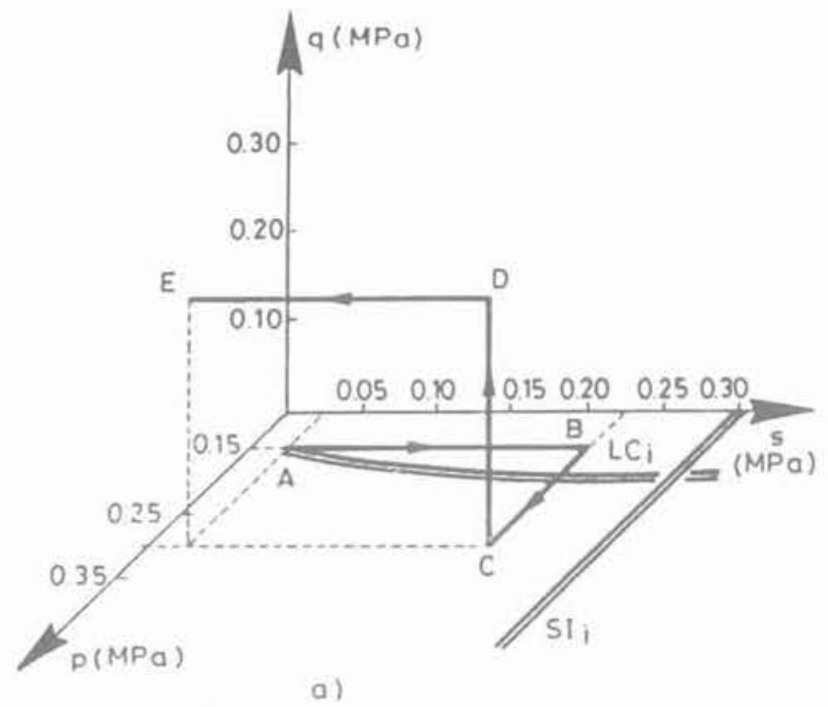

(a) : Trajectoire des contraintes.

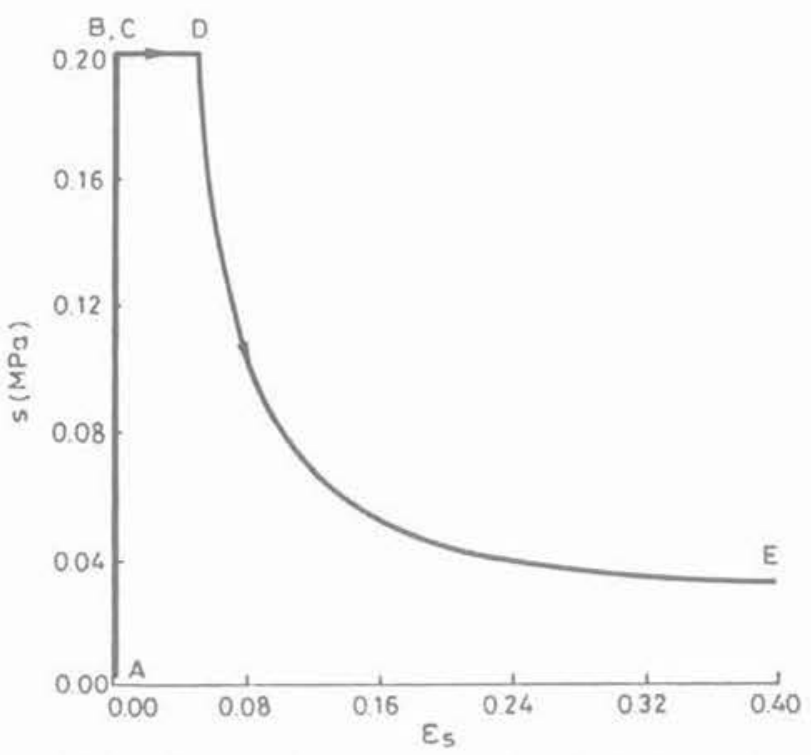

(b) : Relation succion - déformation de cisaillement.

Fig. 13. - Réponse du modèle à un essai provoquant la rupture en humidifiant l'échantillon.

Fig. 13. - Response of model to a test in which failure is induced by decreasing suction (wetting).

\subsection{Remblais faiblement compactés}

Les matériaux faiblement compactés sont grandement instables si pour une raison quelconque leur teneur en eau s'élève. Un cas intéressant de cette réaction se produit lors de remplissage des mines à ciel ouvert à cause de l'extension et de la profondeur que peuvent atteindre ces remplissages. Il peut parfois être nécessaire de construire des bâtiments et des voies de communication sur ceux-ci. Dans ces remplissages, il est fort probable que la teneur en eau augmente soit parce que les niveaux phréatiques retournent à leur position initiale, soit à cause des précipitations.

CHARLES et al. (1984) ont décrit en détail l'effet de l'élévation de la nappe phréatique sur un remblai de fragments de grès et de limonite de $70 \mathrm{~m}$ d'épaisseur. Après avoir installé des extensomètres dans toute la profondeur, la déformation du remblai a été suivie pendant les trois ans qu'a duré l'ascension du niveau de l'eau. Le remblai, comme il arrive souvent dans certains cas, était très hétérogène : la porosité variait entre 0,2 et 0,4 et le degré de saturation initial entre 0,1 et 1 . Les frag. ments de roches tendres prédominaient et le contenu en fines limoneuses était de l'ordre de $10 \%$. Les mesures de tassement effectuées sur l'un des sondages instrumentés sont reproduites sur la figure 14. On y observe la progression des tassements à plusieurs profondeurs au fur et à mesure que le niveau phréatique s'élève. La perméabilité du remplissage est élevée, supérieure à $10^{-4} \mathrm{~m} / \mathrm{s}$.

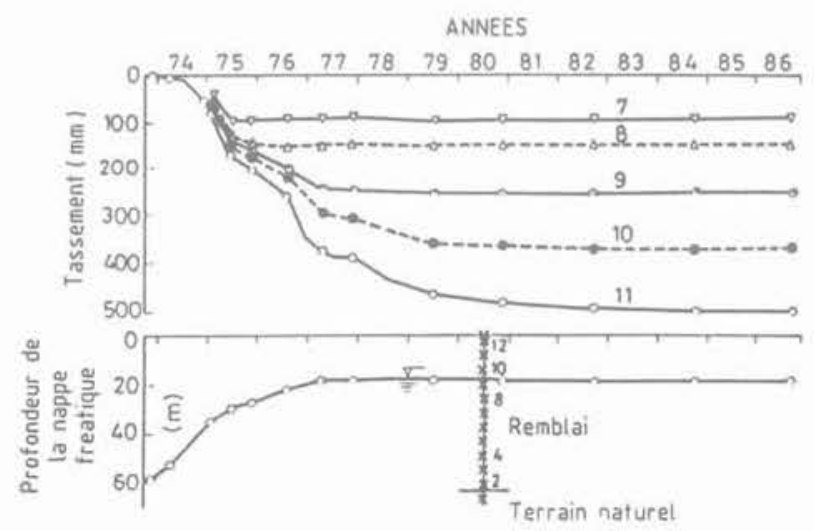

Fig. 14. - Tassements mesurés à Horsley (extensomètre B2) (Charles et al., 1984).

Fig. 14. - Settlements measured at Horsley (extensometer B2) (Charles et al., 1984).

Les coordonnées des tassements représentées par CHARLES et al. (1984) ont été réinterprétées. On a représenté la déformation à différentes profondeurs (c'est-à-dire à des contraintes de confinement différentes) en fonction de la distance entre la surface libre et le point, entre deux bases d'extensomètres séparées de $6 \mathrm{~m}$, représentatif de la déformation. Cette distance, $\mathrm{D}$, est une mesure de la succion existante au point de référence. Lorsque $\mathrm{D}$ se rapproche de zéro, la moitié de la distance séparant deux marques d'extensomètres se trouve submergée. Les résultats apparaissent sur la figure 15 . On retrouve certains phénomènes mis en évidence en laboratoire: si la contrainte de confinement est faible, la diminution de la succion (c'est-à-dire la plus grande proximité du niveau phréatique) peut provoquer des gonflements (c'est le cas de la courbe correspondant à $50 \mathrm{kPa}$ à contrainte verticale moyenne). Si la contrainte moyenne augmente, l'effondrement (courbe de $160 \mathrm{kPa}$ ) augmente aussi. Au-dessus d'une contrainte verticale donnée, le tassement par effondre. ment peut diminuer, étant donné que la compression mécanique elle-même a suffisamment réduit la porosité pour pouvoir limiter le potentiel d'effondrement ultérieur. On observe aussi sur la figure 15 que la majeure partie de l'effondrement se produit pendant la dernière étape de réduction de la succion, lorsque ses valeurs sont déjà faibles. On constate aussi ce phénomène en laboratoire lors des essais à succion contrôlée.

Avec les coordonnées de deux sondages instrumentés on a aussi dessiné les déformations finales en fonction 


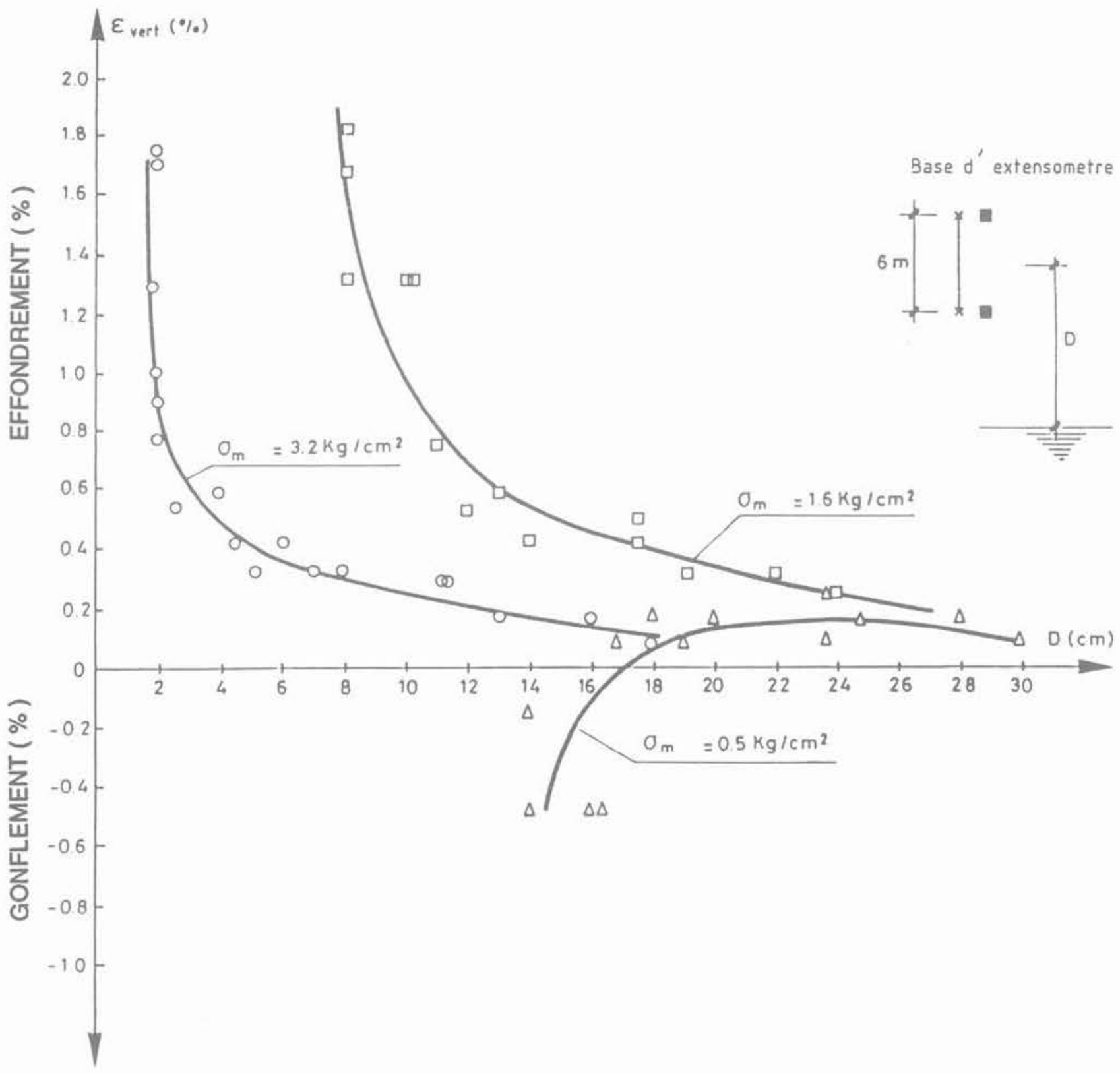

Fig. 15. - Relation entre la déformation d'effondrement (ou de gonflement), la contrainte verticale et la distance à la nappe phréatique, à partir des données publiées par Charles et al. (1984) pour l'emplacement B-2.

Fig. 15. - Relationship among collapse (or heave) deformation, vertical stress and distance to phreatic surface, derived from data published by (Charles et al, 1984) at location B-2.

de la contrainte verticale moyenne. On obtient (fig. 16) une courbe d'effondrement avec un maximum bien marqué et une certaine dispersion pour des contraintes élevées. Un des extensomètres (D-1) correspond à une zone qui a été surchargée par plus de $30 \mathrm{~m}$ de hauteur de remplissage décapés ultérieurement. Dans ce cas deux symboles ont été utilisés pour représenter la contrainte verticale en tenant compte de ce préchargement. On constate que le préchargement réduit effectivement le potentiel de l'effondrement.

Il est évident aussi que la déformation moyenne du remplissage est sensiblement inférieure à la déformation maximale qui peut se produire par un des tronçons intermédiaires. Dans un recueil de cas de tassements provoqués par l'eau dans des remblais de mines à ciel ouvert, CHARLES et BURFORD (1987) trouvent que les déformations moyennes varient entre $0,2 \%$ et $2,5 \%$ (avec une moyenne se situant autour de 1,5\%). Cependant, les valeurs maximales de déformation peuvent être sensiblement supérieures $(5-6 \%)$. Tous ces cas montrent que, s'ils sont humidifiés, soit par remontée des nappes phréatiques soit par infiltration superficielle, les remplissages peu compactés se tasseront de façon significative. Le préchargement est un processus efficace pour limiter ces tassements par effondrement. 


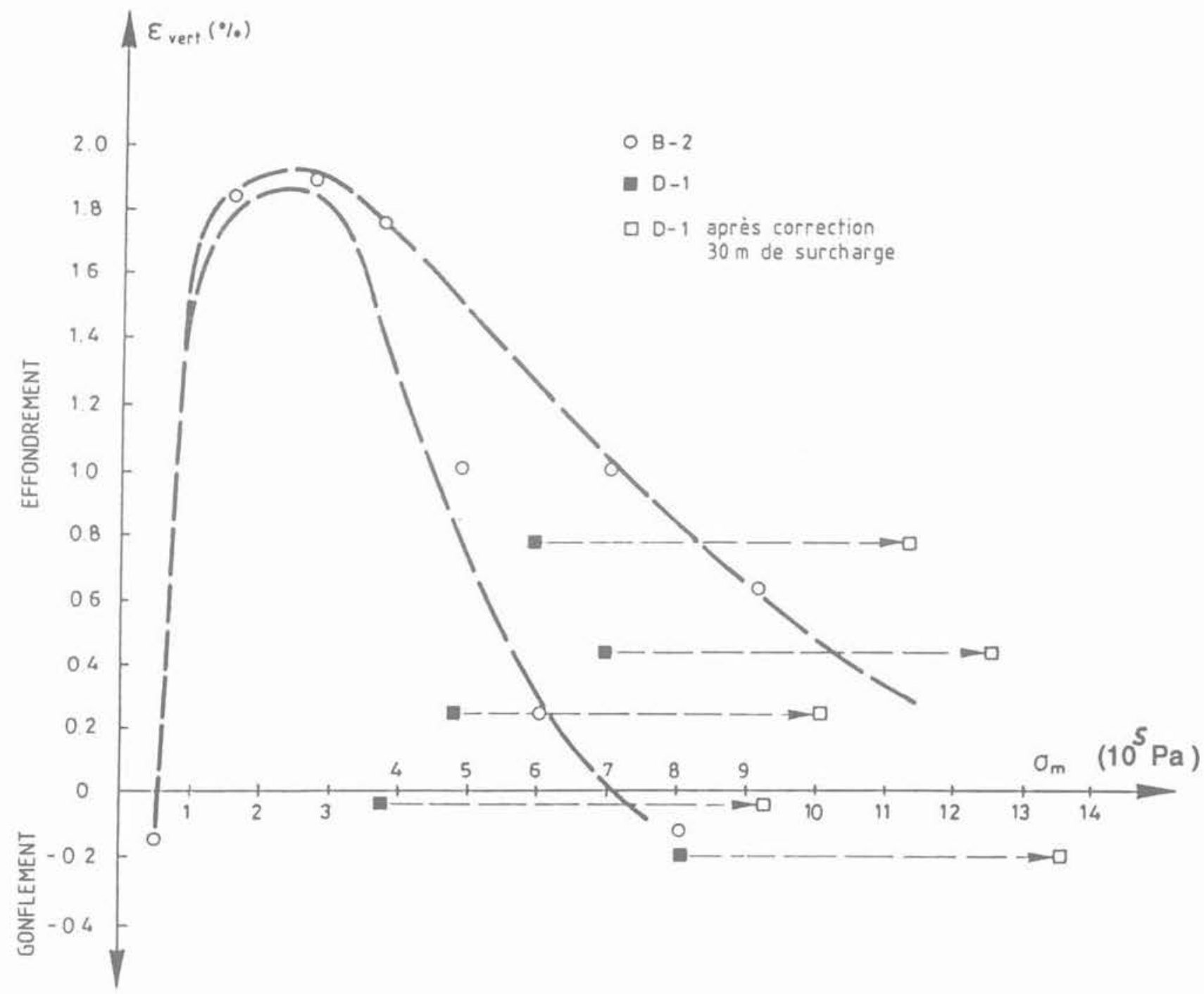

Fig. 16. - Relation entre la déformation volumique et la contrainte moyenne calculée en fonction des données publiées par (Charles et al., 1984).

Fig. 16. - Relationship between volumetric deformation and mean stress computed from data published by (Charles et al. 1984)

\subsection{Remblais de marnes argileuses compactées}

Les roches tendres argileuses présentent certainement les plus grandes difficultés de réemploi en remblai. Les éventuelles conséquences de deux philosophies d'exécution illustrent bien les difficultés :

a) $\mathrm{Si}$ l'on tasse énergiquement avec un compactage maximal, il est fort probable qu'une humidification ultérieure se traduise par des gonflements, surtout sur des surfaces non protégées (talus et leur couronnement). De plus, le compactage énergique peut rompre les liaisons diagénétiques des fragments de roche et $\alpha$ libérer * un plus grand potentiel expansif.

b) En revanche, si le remblai se tasse en couches de plus grande épaisseur sous son propre poids, des vides favorisant l'effondrement apparaitront dans la masse. A la longue, la dégradation des blocs contribuera au tassement.
Trouver un juste équilibre est probablement très difficile. Outre les conditions de compactage, les mesures complémentaires limitant les arrivées d'eau au remblai seront fondamentales.

Les remblais construits avec ces matériaux évolutifs pré. sentent généralement des mouvements et des déformations au cours du temps se traduisant par l'apparition de fissures sur la chaussée (spécialement à proximité des accotements où l'intensité des changements cycliques de l'humidité est la plus grande), de tassements localisés, de reptations et de glissements superficiels dans les talus du remblai et, dans des cas extrêmes, de rupture généralisée.

Tous ces problèmes sont particulièrement bien illustrés dans le comportement d'un remblai expérimental de marnes argileuses réalisé dans le Sud de la France (MIEUSSENS et CAMAPUM de CARVALHO, 1986; MIEUSSENS, 1989). La dense instrumentation installée 
a permis de connaitre de façon détaillée l'évolution du remblai.

Il s'agissait d'un remblai de $30 \mathrm{~m}$ de hauteur (remblai de LA JORLE) construit avec des marnes argileuses $\left(\mathrm{CO}_{3} \mathrm{Ca}: 70-80 \% ; w_{1}: 14-18 \% ; w_{\text {nat }}: 9-11 \%\right)$ compacté à des densités variant entre les optima du Proctor Normal et du Proctor Modifié. Le degré de saturation moyen après compactage était de l'ordre de $90 \%$.

Des essais d'imbibition sur des échantillons compactés à l'optimum normal et modifié (fig. 17) montrent qu'à des densités correspondant à l'OPM le matériau peut gonfler d'une maniëre importante si le confinement est faible, alors qu'à des densités correspondant à l'OPN le risque d'effondrement à des contraintes croissantes est plus grand (il semble cependant que ces essais sous: estimèrent la pression réelle de gonflement).

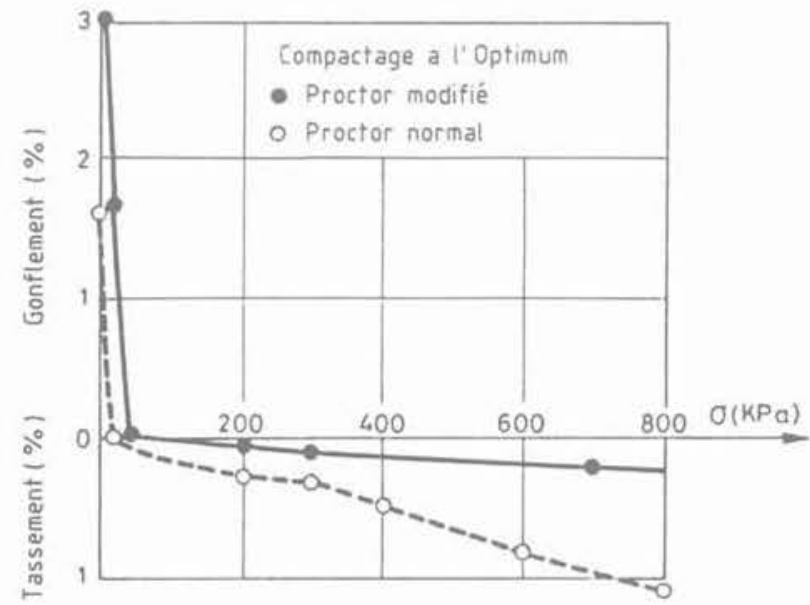

Fig. 17. - Influence de la saturation sur le gonflement ou l'effondrement en fonction de la pression initiale de consolidation (Mieussens et Camapum de Carvalho, 1986).

Fig. 17 - Effect of saturation on swelling or collapse deformation as a function of initial consolidation pressure (Mieussens and Camapum de Carvalho, 1986).

Sur un matériau relativement imperméable, comme par exemple la marne compactée, les changements d'humidité se produisent surtout dans les couches superficielles non protégées et soumises à l'action cyclique de l'infiltration et de l'évaporation atmosphérique. Il y a peu de résultats expérimentaux sur l'effet des cycles de succion sur la déformation d'une argile compactée, mais certaines données disponibles mettent en évidence une accumulation progressive du gonflement. Comme le montre la figure 18 où les résultats d'essais œdomé. triques à succion contrôlée sur un échantillon d'argile de Madrid, gonflante et compactée, sont présentés (POUSADA, 1984). Le gonflement significatif créé par la première humidification ne cesse d'augmenter au cours des cycles suivants de séchage et d'humidification.

Dans le cadre du remblai de La Jorle, un extensomètre continu placé à mi-hauteur du versant montra, après les tassements de la phase de construction, un gonflement important des deux derniers mètres du remblai. Paral-

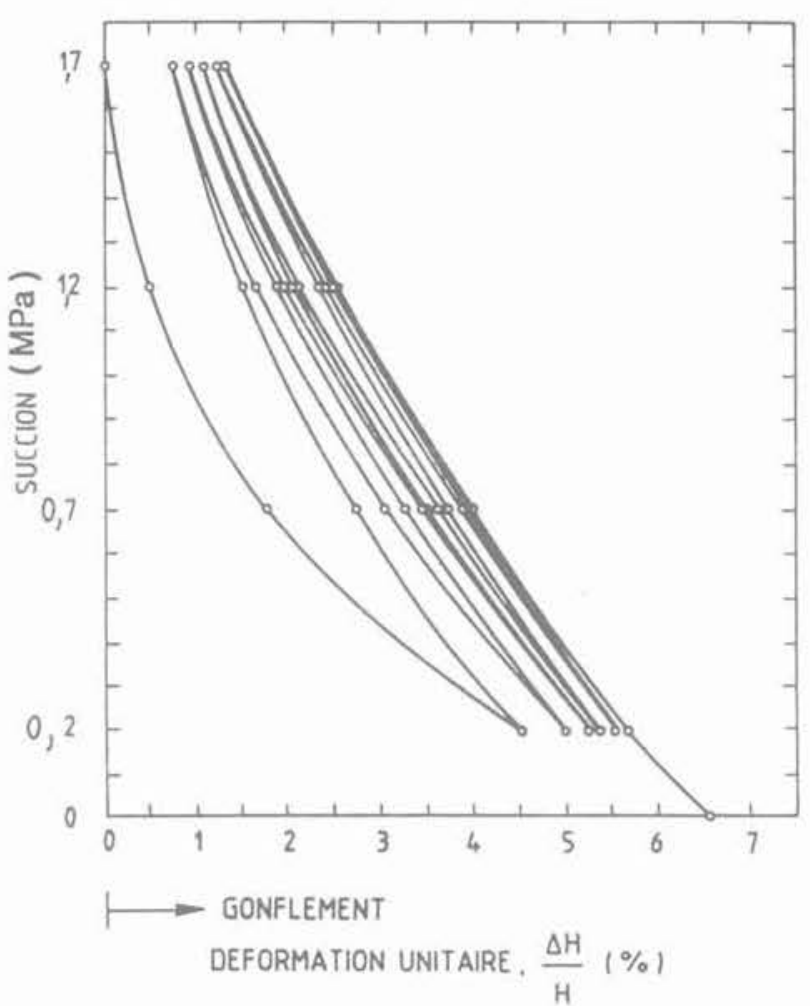

Fig. 18. - Changement de volume dû aux cycles de succion $\left(W_{0}=24 \% ; \sigma_{v}=10 \mathrm{Kpa}\right)$, (Pousada, 1984).

Fig. 18. - Volume changes induced by suction cycles.

lèlement, un inclinomètre placé au même endroit détecta des mouvements horizontaux au bas du versant, progressivement croissants, sur les trois derniers mètres du remblai.

L'évolution des deux composantes du mouvement est très bien rendue sur la figure 19. Les premières phases du mouvement correspondent presque entièrement à une déformation purement volumique d'une certaine épaisseur du talus. Le vecteur déplacement est pratiquement normal au talus, mais à mesure que le temps s'écoule, ce sont les mouvements dans le sens du talus qui prédominent, indiquant la présence d'importantes déformations de cisaillement pouvant conduire, à plus longue échéance, à des ruptures superficielles causées par des contraintes de cisaillement.

Certains essais mesurant les contraintes dans des sondages réalisés dans le talus à des dates différentes confirment la dégradation progressive de la résistance du terrain. Une couche "active » apparaît en surface dont l'épaisseur croît avec le temps. Le modèle de comportement ébauché au paragraphe 2.1 aide à interpréter les phénomènes qui résident dans le talus du remblai. En effet, sur la figure $20 \mathrm{a}$ est montré qualitativement l'état de contrainte $(p, q)$ d'un point de marne représentatif, proche du talus (point A) à la fin de la construction. Sur la figure, est aussi indiquée la surface d'écrouissage correspondant à cet état initial. Le sol n'a encore souffert d'aucune humidification, et il est probable que le point $A$ se trouve dans le domaine élastique. Au fur et à mesure que l'humidification avance (fig. $20 \mathrm{~b}$ et c), la succion ainsi que la surface d'écrouis- 


\section{DATES DES MESURES}

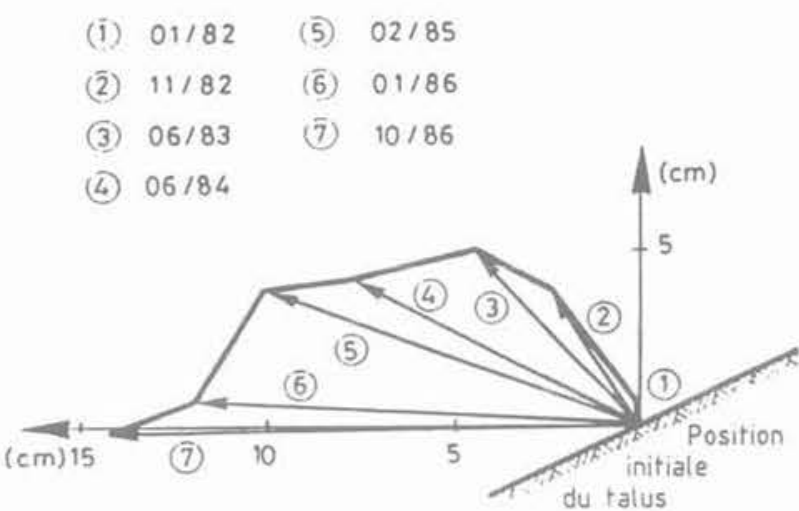

Fig. 19. - Mouvement de la surface du talus déduite de la composition des déplacements horizontaux et verticaux mesurés à différentes dates (Mieussens, 1989).

Fig. 19. - Surface displacements at the slope derived from the combination of horizontal and vertical displacements measured at different dates (Mieussens, 1989).

sage diminuent. Dans la première étape (dans le domaine élastique), la réduction de succion se traduit uniquement par des déformations volumiques de gonflement. Mais dès que la surface d'écrouissage est atteinte (fig. 20b) des déformations irréversibles de volume et de cisaillement se produisent. Une humidification supplémentaire (fig. 20c) renforce la composante de déformation irréversible de cisaillement pouvant même mettre le sol dans des conditions critiques. Ce cas serait similaire à celui de la figure 12 . Il montre la possibilité d'atteindre la rupture par humidification.

Dans ce cas là, il faudrait ajouter aux phénomènes dé. crits, la dégradation des propriétés de la marne compactée, non attribuable exclusivement aux changements de succion. Ce second facteur de dégradation subie par la marne aurait plusieurs origines: les propres déforma. tions irréversibles (plastiques) du matériau et les phénomènes d'origine physico-chimiques.

Ce cas est particulièrement instructif pour proposer certaines mesures améliorant le comportement des rem. blais de sols et de roches argileuses:

- protection des talus (et en général de tout l'environnement extérieur) face à des changements d'humidité. Les bermes sont déconseillées dans ce cas-là, les pentes uniformes étant préférables ;

- réduction des contraintes de cisaillement dans les talus. Le procédé est de réduire sa pente. De cette façon on réduit la tendance aux déformations par cisaillement lors des changements d'humidité ;

- zonage du remblai de façon à ce que le compactage soit plus intense dans ses zones les plus confinées. Autre alternative, installer des matériaux plus sélectionnés, insensibles aux changements d'humidité, dans l'environnement extérieur du remblai ou sous la forme de couches de type "sandwich * si l'humidité des matériaux argileux est très élevée (RODRIGUEZ MIRANDA et al., 1986).

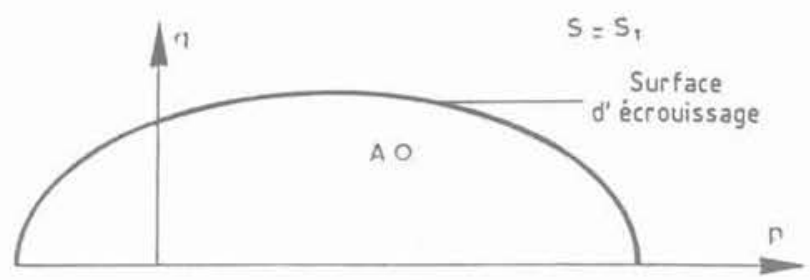

a)

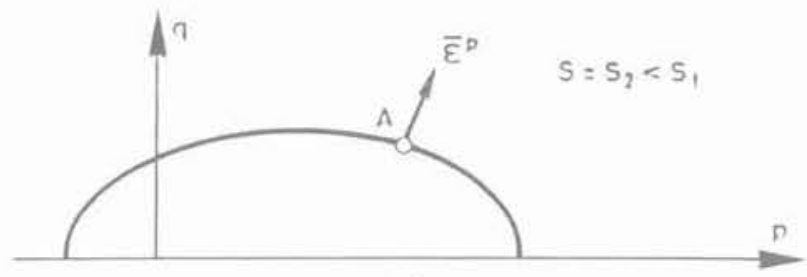

b)

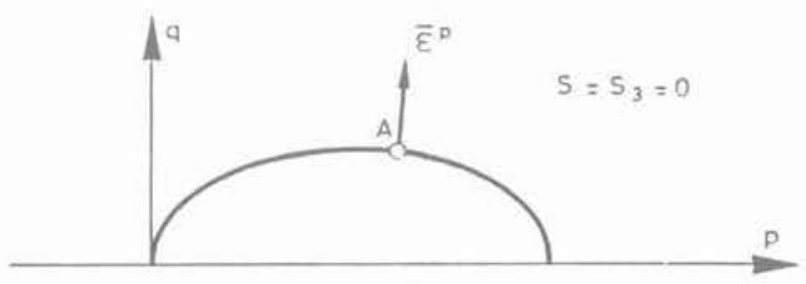

c)

Fig. 20. - Illustration de l'évolution de l'état de contrainte d'un point proche de la surface du talus.

Fig. 20. - Illustration of the evolution of stress state of a point close to the slope surface.

\section{CONCLUSIONS}

Dans cet article nous avons examiné les problèmes de déformation causés par les variations de teneur en eau au cours de la période d'utilisation des remblais. Pour cela nous avons décrit les traits principaux du comportement des sols compactés à partir d'essais de laboratoire et expliqué certains cas réels significatifs. Les principales conclusions sont les suivantes:

1) Les sols compactés peuvent gonfler ou s'effondrer suite à une augmentation de teneur en eau en fonction de leur hurnidité, de leur minéralogie, de la densité après compactage et des contraintes appliquées. Pour un sol compacté loin de la saturation, la densité semble être la variable qui contrôle fondamentalement son comportement lors d'une humidification. Par ailleurs, l'effondrement en cours d'humidification atteint un maximum à une contrainte déterminée de confinement pour décroître ensuite.

2) Un modèle capable de décrire correctement les changements de volume des sols compactés, sous l'action des contraintes extérieures et des changements de succion, a été introduit. Le modèle prend en compte les efforts et les déformations de cisaillement. Il est utile pour interpréter certains mécanismes de déformation produits à proximité des talus des remblais.

3) L'analyse de la saturation de remblais peu compactés, utilisés dans le remblaiement des mines à 
ciel ouvert, a permis de valider certaines de ces propriétés mesurées en laboratoire:

- l'effondrement atteint un maximum pour une contrainte moyenne, mais se transforme en gonflement à de faibles contraintes de confinement ;

- l'effondrement est un processus graduel contrôlé par l'intensité de la succion (mesurée dans ce cas-ci comme distance à la position de la surface libre).

4) Les résultats d'une instrumentation abondante, installée dans un remblai de mames compactées, ont permis de suivre les étapes successives de la déformation des talus: après une première étape de gonflement isotrope, d'importantes déformations de cisaillement se développent, favorisées par la progressive diminution de la succion initiale et par la propre dégradation du ma. tériau. Au dernier stade, la rupture par un effort de cisaillement du matériau compacté peut se produire. Le modèle de comportement développé est compatible avec ces observations et donne la possibilité d'en faire une analyse plus précise dans le futur.

\section{Remerciements}

Au cours de la préparation de ce travail nous avons demandé des renseignements à certaines personnes connaissant et analysant les thèmes abordés. Nous les remercions toutes pour leur cordiale réponse et leur collaboration: V. ESCARIO du Laboratoire de Carreteras y Geotecnia de Madrid (Espagne), J.A. CHARLES du Building Research (Royaume-Uni), E. MARANHA DAS NEVES du Laboratorio Nacional de Engenharia Civil de Lisbonne (Portugal) et P. DELAGE de l'Ecole Nationale des Ponts et Chaussées de Paris. Nous remercions aussi la collaboration de M. A. BALMACEDA, F. BATTLE, A. GENS et A. JOSA du département de Ingenieria del Terreno de l'ETS d'Ingenieros de Ca. minos, Canales y Puertos de Barcelone (Espagne).

\section{BIBLIOGRAPHIE}

ABEYESEKERA R.A., LOWELL C.W. and WOOD L.E. (1979), Stress-deformation and strength characteristics of a compacted shale. Clay Fills, ICE, pp. 114.

AITCHISON G.D. and MARTIN R. (1973), A membrane oedometer for complex stress-path studies in expansive claus. Proc. 3rd Int. Conf. Expansive Soils, Haifa, 1, pp. 161-167.

ALONSO E.E., GENS A. and HIGHT D.W. (1987), Special Problem Soils. General Report, Proc. 9th ECSMFE, Dublin.

ALONSO E.E., GENS A. y JOSA A. (1990), A constitutive model for partially saturated soils. Geotech. nique $40, n^{\circ} 3$, pp. $405-430$.

BALMACEDA A. (1991), Suelos compactados. Un estudio teorico y experimental. Tesis Doctoral, ETS Ingenieros de Caminos, Barcelona.

BARDEN I., MADEDOR A.O. and SIDES G.R. (1969), Volume change characteristics of unsaturated clays. J. Soil. Mech. fd. div. ASCE 95 (SM1), pp. 33-51.

BRACKLEY I.J.A. (1975), A model of unsaturated clay structure and its application to swell behaviour.
Proc. 6th. Reg. Conf. for Africa on SMFE, Durban, 1 , pp. 71.79.

CHARLES J.A., HUGHES D.B., BURFORD D. (1984), The effect of a rise of water table on the settlement of backfill at Horsley restored opencast coal mining site 1973-1983. Proc. 3rd. Int. Conf. on Ground Movements and Structure, pp. 423-442.

CHARLES J.A. and BURFORD D. (1987), Settlement and groundwater in open-cast mining backfills. Proc. 9th ECSMFE, Dublin, 1, pp. 289-292.

COX D.W. (1973), Volume change of compacted clay fill. Clay Fills, ICE, London, pp. 79-86.

DAPENA E. (1989), Efecto de la compactación sobre el comportamiento frente al colapso de un mate. rial arcilloso. Simposio el agua y el terreno en las infraestructuras viarias, Torremolinos.

DELAGE P. and SURAJ DE SILVA G.P.R. (1992), Suction controlled testing of non saturated soils with an osmotic consolidometer. 7th International Conference on Expansive Soils, Dallas, pp. 206211.

DELGADO (1986), Influencia de la trayectoria de las tensiones en el comportamiento de las arcillas expansivas y de los suelos colapsables en el laboratorio y en el terreno. Tesis Doctoral, Universidad de Sevilla.

ESCARIO V. (1967), Measurement of the swelling characteristics of a soil fed with water under tension. Int. Coop. Res. on the Prediction of Moisture Content under Road Pavements, Working Group under the auspices of OCDE, Madrid Meeting.

ESCARIO V. and SAEZ J. (1973), Measurement of the properties of swelling and collapsing soils under controlled suction. Proc. 3rd. Int. Conf. Expansive Soils. Haifa, pp. 196-200.

ESCARIO V. (1987), Terraplenes y Pedraplenes. Direccion General de Carreteras, MOPU, Madrid.

FREDLUNG D.G. and MORGENSTERN N.R. (1976), Constitutive relations for volume change in unsaturated soils. Can. Geotech. Jnl. $13(3)$, pp. 261276.

JIMENEZ SALAS J.A. y SERRATOSA J.M. (1953), Compressibility of clays. Proc, 3rd ICSMFE, Zurich, 1, pp. 192-198.

JOSA A., BALMACEDA A., GENS A. and ALONSO E.E. (1992), An elastoplastic model for partially saturated soils exhibiting a maximum of collapse. Third Int. Conf. on Computational Plasticity, Barcelona, Part 1, pp. 815-826.

JUSTO J.L., SAURA J., RODRIGUEZ J.E., DELGADO A. and JARAMILLO A. (1984), A finite element method to design and calculate pier foundations in expansive-collapsing soils. Proc. 5th. Int. Conf. Exp. Soils, Adelaïde, pp. 119-123.

KASSIF G. and BEN-SHALOM A. (1971), Apparatus for measuring swell potential under controlled moisture intake. J. of Materials, ASTM, 6, 1, pp. 3 15.

LLORET A. and ALONSO E.E. (1985), State surfaces for partially saturated soils. Proc. 11th ICSMFE, San Francisco, 2, pp. 557-562.

MASWOSWE J. (1985), Stress path for a compacted soil during collapse due to wetting. $\mathrm{Ph}$. D. Thesis, Imperial College, London. 
MATYAS E.L. and RADHAKRISHNA H.S. (1968), Vo. lume change characteristics of partially saturated soils. Geotechnique, $18(4)$, pp. 432-448.

MIEUSSENS C. y CAMAPUM DE CARVALHO J. (1986), Remblais en Marne. Désordres, étude, confortation. Scétauroute Laboratoires des Ponts et Chaussées, Paris.

MIEUSSENS C. (1989), Influence de l'état hydrique sur le comportement des argiles en remblai. Journées de Physique des Arts. L'eau dans les matériaux. G. Raimbault ed., pp. 131-135.
POUSADA E. (1984), Deformabilidad de arcillas expansivas bajo succion controlada. Tesis Doctoral, Univ. Polit. Madrid.

RODRIGUEZ MIRANDA M.A. (1986), Pedraplenes. Simposio sobre Terraplenes, Pedraplenes y otros Rellenos. Madrid, pp. 219-248.

VICOL T. (1990), Comportement hydraulique et mécanique d'un sol fin non saturé. Application à la modélisation. Thèse de Doctorat, ENPC, Paris.

YUDHBIR (1982), Collapsing behaviour of residual soils. Proc. 7th Southeast Asian Geot. Conf. HongKong, 1, pp. 915-930. 


\title{
JOURNÉES D’ÉTUDE
}

\section{LA MAÎTRISE DES INONDATIONS RAPIDES LIÉES AU RUISSELLEMENT URBAIN ET NATUREL}

\author{
Paris, mercredi 17 et jeudi 18 mars 1993
}

Ces journées d'étude s'adressent aux ingénieurs des services techniques des collectivités territoriales, aux gestionnaires d'ouvrages exposés aux risques d'inondations, aux aménageurs et urbanistes ainsi qu'aux enseignants et chercheurs concernés par la maîtrise de l'eau en tant que risque naturel.

\section{Objectifs-Contenus :}

Des événements spectaculaires viennent périodiquement rappeler l'importance du risque d'inondations dans les bassins versants urbanisés ou naturels. Des efforts importants ont été faits sur certains sites pour essayer de limiter les effets de ces inondations, souvent catastrophiques, et des progrès ont été réalisés au cours des dernières années tant pour la prévision des causes météorologiques des inondations que pour une meilleure conception des dispositifs de protection ou aménagements du paysage qui contribuent à mieux gérer ce risque naturel.

Ces journées ont pour objectif de faire le point sur l'état actuel des pratiques, politiques locales ou moyens techniques concernant ce problème. On y traitera notamment de la prévision des pluies, des moyens techniques permettant de retenir l'eau sur les bassins versants ou au contraire d'en accélérer l'écoulement ou encore de diminuer les impacts d'une crue (par exemple, réglementation du stationnement, déplacement et protection de réseaux), des stratégies mises en cuuvre dans différents pays à l'échelle d'une ville ou d'un départernent, des effets de l'eau sur les pentes (érosion), etc.

\section{LE NOUVEAU GUIDE TECHNIQUE "RÉALISATION DES REMBLAIS ET DES COUCHES DE FORME "}

Paris, mardi 23 mars 1993

Cette journée d'étude s'adresse aux ingénieurs et techniciens dans le domaine de la conception et la construction: maîtres d'œuvre, bureaux d'études, entreprises, bureaux de contrôle.

\section{Objectifs-Contenus :}

Le Nouveau Guide Technique "Réalisation des remblais et des couches de forme " publié en septembre 1992 par le SETRA et le LCPC enrichit et complète la RTR de 1976 qui fixait le cadre mé- thodologique de l'étude et la réalisation d'un projet de terrassement.

L'objet de cette journée est de permettre aux participants :

- d'appréhender la teneur des modifications et des aspects novateurs introduits dans ce document,

- d'en mesurer les incidences sur l'étude et la réalisation des ouvrages en terre et des couches de forme, qu'ils soient on non déjà familiarisés avec la démarche RTR 1976.

Le Congrès GEOCONFINE orgnanisé par le CFGI et le BRGM et présidé par M. MOUSEL (ADEME) se tiendra à Montpellier (France) du 8 au 11 juin 1993.

Pour tous renseignements s'adresser au secrétariat :

\author{
Michel BARRES \\ BRGM \\ Dépt. "Environnement » \\ BP 6009 \\ 45060 ORLÉANS CEDEX - France \\ Tél. : (33) 38.64.34.14.
}

